\title{
Overcoming Impasses in Conversations: A Creative Business*
}

\section{Ilaria Torre}

Plymouth University, UK

E-mail address: Ilaria.Torre@Plymouth.ac.uk

\section{Frank Loesche}

Plymouth University, UK

E-mail address: Frank.Loesche@Plymouth.ac.uk

\section{ARTICLE INFO}

\section{Keywords:}

Impasse

Conversation

Creative problem solving

Conversation Analysis

\section{Article history:}

Received: 13 March 2016

Received in revised form: 29 July 2016

Accepted: 15 October 2016

ISSN: 2354-0036

DOI: $10.1515 /$ ctra-2016-0016

\section{A B S TRACT}

The ability to communicate complex meanings is a specific human ability which plays a crucial role in social interactions. A habitual example of these interactions is conversation. However, we observe that spontaneous conversation often hits an impasse when none of the interlocutors immediately produces a follow-up utterance. The existence of impasses in conversations, and the way that interlocutors overcome them provide evidence for our argument that conversation is a sequence of creative problem solving. In this work we use techniques from Conversation Analysis (CA) on publicly available databases of naturally-occurring speech and we suggest a framework to understand how impasses are reached and overcome. As a result, we hope to reveal yet another instance of the bond between language and creativity.

\section{INTRODUCTION}

In this paper we discuss conversations between humans and explore the possibility of interpreting them as continuous creative problem solving, where the problem is represented by the common phenomenon of impasse.

\section{Conversation}

While scholars have not reached an agreement on a formal definition of conversation, it is at least possible to isolate the phenomenon according to the elements associated with it. A conversation is often referred to as a meaningful verbal communication between at least two participants (Donaldson, 1979), who cooperatively construct an interaction (Clark, 1996; Warren, 2006). Ritualized exchanges as well as interactions between obvious status differences are often excluded from the definitions (Donaldson, 1979; War-

\footnotetext{
* This work was supported by CogNovo (FP7-PEOPLE2013-ITN-604764), a project funded by the EU Marie Curie programme. The authors wish to thank Dr. Traci Walker for her suggestions on the conversation corpus
} 
ren, 2006). In this sense, interactions such as greetings, lectures, or orders are not usually considered conversations. Conversation generally serves the purpose of creating and maintaining social relationships (Donaldson, 1979; Kaplan, 1997; Sacks, Schegloff, \& Jefferson, 1974). Its social and collaborative nature can also be evinced by one of its most prominent and necessary features, turn-taking. If interlocutors were not able to follow the implicit rules of turn-taking, it would not be a conversation, but rather a chaotic ensemble of sounds. Even though the content of a conversation might not be an information exchange, the fact that each utterance is an action which implies something is a well-known theory in Philosophy of Language (Austin, 1975; Grice, 1957, 1975; Searle, 1969). From this point of view, we can say that conversations are meaningful because, even though they might not convey any new information to the interlocutors, they mean something for all of them, and they can be the means by which a change in the environment takes place (e.g. someone says "I'm cold" followed by another person closing the window). Another element associated with conversation and that distinguishes it, for example, from written communication is improvisation: interlocutors in a conversation do not know in advance what others or themselves will say in the next turns, so they need to be able to - as Donaldson puts it - creatively adapt to it (Donaldson, 1979; Halliday, 1994; McCarthy, 2001). Thus, following the summary in Jordan et al. (2009), we can conclude that conversation is made up of three main components: social collaboration, meaningfulness and improvisation.

\section{Problem Solving}

Problem solving can be seen as a way of reaching a goal. A problem can be well-defined, i.e. having an expected solution, a clear goal, and a defined path on how to reach it. Lacking any of these elements makes it ill-defined. However, the boundary between these types of problem does not seem to be easily distinguishable (Reed, 2015; Simon, 1973). Creative problem solving adds a notion of creativity to it: Runco \& Jaeger emphasize that a creative solution has to meet the minimal requirement of being novel work that is accepted as tenable or useful or satisfying by a group in time, as initially put forward by Stein (Runco \& Jaeger, 2012; Stein, 1953). Often a third attribute of surprise is considered to be compulsory (Boden, 2004). These three attributes have different names in the creativity and creative problem solving literature, either using synonyms or similar but not equal terms. In social situations additional elements are frequently mentioned as identifiers of creativity - such as expression - while usefulness might be referred to as social appreciation as a result of social evaluation (Fischer, Giaccardi, Eden, Sugimoto, \& Ye, 2005). Taking the personal-psychological creativity of the individual into account (see Boden, 2004), a distinction between convergent and divergent thinking, following 
Guilford, is helpful: convergent tasks have one possible solution while divergent thinking might arrive at several correct solutions (Guilford, 1967). Both processes have been linked to problem solving, for example as a search within a problem space (e.g. Newell \& Simon, 1972), or as a divergent production of potential solutions (Gilhooly, Fioratou, Anthony, \& Wynn, 2007).

\section{Conversation as Creative Problem Solving}

In the rest of the section we explore similarities between conversation and creative problem solving. As we have seen, conversation includes elements of social collaboration, meaningfulness, and improvisation, while creative problem solving in a social situation can be described as a novel, expressive, and useful way of reaching a goal. In problem solving this goal does not have to be tangible, e.g. for ill-defined problems. Just as in Fischer et al.'s (2005) description of social creativity people need to express their ideas, interlocutors articulate their utterances in a conversation - they express them. Similarly, the other attributes mentioned above show a large potential overlap between conversation and creative problem solving: meaningfulness, as described by Jordan et al. (2009), shows some similarity to the idea of usefulness. Improvisation, either as an acquired skill or a constrained combinatorial creation, includes subjective novelty in this context. Since conversations usually do not aim at a clearly defined goal, and since their structure is also not defined, they show similarities to ill-defined problems. Similarly, Demuth and Glaveanu (2016) argue that spontaneity and creativity contribute to the construction of utterances based on predictions of what the other person might say in the future; in this sense, conversation can also be understood as a problem finding approach in an illdefined environment.

The link between creativity and conversation can be observed on a more granular level as well. For example, Kaufman and Beghetto (2013) distinguish between subjective self-discovery (mini-C), everyday creativity (little-c), and geniuses (big-C). Between and within each of these groups different tasks might require various time spans to be solved. Similarly, a longer conversation can consist of smaller parts, each of which can be dedicated to a certain topic or carry a distinguishable meaning. Each of these sub-parts shows the same previously highlighted characteristics of the whole conversation: it is a social collaboration, which includes novelty and improvisation, and it is useful by being meaningful for the participants. As these sub-parts sequentially follow one another, eventually either another sub-part is initiated or the whole conversation is terminated after the end of a sub-part. We should mention that we are not considering the ability to create new linguistic symbols, which is another instance of creativity in language, but we only focus on the process of overcoming impasses. 


\section{Impasse}

Creative problem solving, or more generally creativity, is often described using a sequential model similar to the famous one proposed in Wallas (1926). He suggested that creative problem solving can be understood as a sequence of phases which he named preparation, incubation, illumination, and verification. A following phase can only start if the previous one is completed. Even though the number and nature of these sequential steps has been the subject of debate for the past 90 years, the idea of using sequential or componential models has proven to be a valid way of describing the creative process in individual as well as social contexts (also see Amabile, 1983). Howard, Culley, \& Dekoninck (2007) compare 42 models, more than 20 of which present a sequence of phases similar to those previously mentioned. A situation within this sequence, where one does not know how to proceed, is referred to as an impasse (Cranford \& Moss, 2012). An impasse is therefore, at least from an external point of view, accompanied by the passing of time, without any advancements in the process, or, to use terms that are found in conversation studies, a gap or lapse (Sacks, Schegloff, \& Jefferson, 1974). Consequently, all the sequential models mentioned by Howard, Culley, and Dekoninck (2007) include a concept similar to that of an impasse.

\section{Framework}

In this section we want to offer a more detailed speculation on what might happen during a conversation regarding impasses, but reduce our explanation to the elements of a conversation necessary to follow this article. Since elements of our model relate to Conversation Analysis (CA) as well as creative problem solving, we avoid technical terms in order not to confuse them. In spoken communication silences are intentionally used as a rhetorical instrument and to prevent or even provoke turn-taking, but they also occur unplanned by the interlocutors. In this paper we focus on the latter case and refer to these occurrences as impasses.

To start with some examples: during a conversation, a difficult and open-ended question is posed, but no-one has an answer to it, or a topic in a conversation has been exhausted. In both cases, the interlocutors might have difficulties finding the next meaningful and non-repetitive thing to say. If one of them starts a follow-up topic early enough, the others might still have experienced the impasse, even though it might have gone unnoticed by an external listener. On the other hand, if none of the speakers is able to proceed, this impasse produces an unnaturally long silence - what Sacks, Schegloff, and Jefferson (1974) call a lapse. We argue that these pauses can be observed by an outside listener, especially since Stivers et al. (2009) have shown universal patterns in response 
latency, i.e. silences, across languages and cultures. From a probabilistic point of view, the observable impasses are more likely to happen with a low number of interlocutors, with the additional benefit of having a simpler overall communication structure.

To illustrate the context of an impasse happening within a 2-interlocutor conversation, we refer to Figure 1.

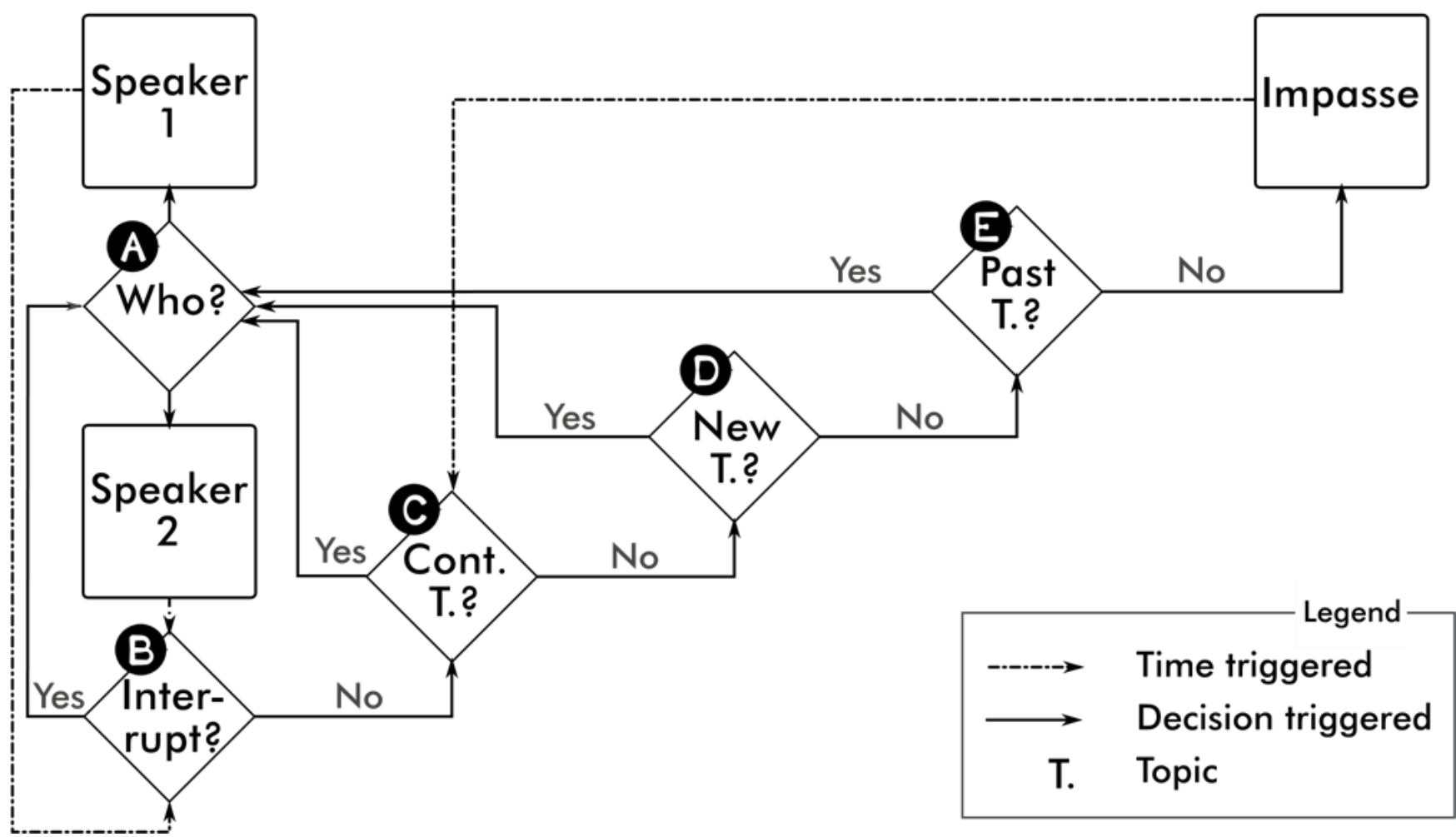

Figure 1 Impasses in conversations

All the square boxes represent time-based events that can be observed from an outside perspective. One of them represents an impasse, as we hypothesize that solving it takes considerable time that can be observed as an increased response latency by an outside observer. The passing time is emphasized in our figure by the time triggered transitions to one of the decisions, represented by diamond shapes (e.g. B). These decisions, on the other hand, happen quickly between interlocutors (e.g. A and B), or internally to one speaker ( $\mathrm{C}$ to $\mathrm{E})$ - too fast to be identified by outside observers. Notice that, as ritualized communication is excluded from our analysis, following the definitions described in the Introduction, elements such as greetings and closing remarks are not included in the diagram. Step A represents the moment when either Speaker 1 or Speaker 2 is starting to talk. For an extensive discussion on this step and the processes involved, we refer to Sacks, Schegloff, and Jefferson (1974), Selting (1996), and Stivers et al. (2009). For our model it is sufficient to have a step $A$ which results in one speaker starting to talk. Once 
this decision is made, the active speaker talks for a certain amount of time, checking for cues of being interrupted by the other speaker at B. This can happen very quickly, as shown by Chambliss and Feeny (1992), and Cummins, Li, and Wang (2013). If the speaker understands that he / she is being interrupted, the turn is either taken by the other speaker or the same speaker continues at A. In the latter case and if the topic is not yet depleted and can be continued $(C)$, the conversation is continued at $A$. If the topic cannot be continued at $\mathrm{C}$ and one of the speakers has a new topic ready, the conversation continues from $D$ to $A$. The conversation also continues in the case of one of the speakers deciding to revisit a previous topic at $\mathrm{E}$. If this is not the case either, the conversation does not progress and arrives at an impasse. During the impasse a speaker might re-evaluate the possibility of either continuing the current topic at $C$, starting a new one at $\mathrm{D}$, or going back to a previous topic at E. Participants might iterate through these possibilities for some time before one of them is able to start talking again at A. If the step from $C$ to $D$ is iterative and stays within the semantically constrained problem space, these loops are similar to the search described in Newell and Simon (1972).

A large semantic step or conceptual leap in finding a new topic could be described as a restructuring process and could therefore be identified as an insight (Beeftink, Van Eerde, \& Rutte, 2008; Öllinger, Jones, \& Knoblich, 2014; Salvi, Bricolo, Kounios, Bowden, $\&$ Beeman, 2016). While the order and the number of iterations of these steps can differ between individuals and between impasses in the same conversation, this model assumes that both interlocutors will try to overcome the impasse. However, in other cases the impasses can also mean the end of the conversation, with the interlocutors switching to a ritualized closing remark from step $A, C, D$, or $E$.

If conversation is a continuous stream of problem solving, steps C, D and E represent the ill-defined problem of either continuing with the current topic, choosing a new one, or going back to a previous one. In our framework these steps are also processed sequentially, even though the exact order might be different for each problem. In terms of novelty and usefulness, it could be argued that each meaningful unit produced within a topic is non-repetitive. Step $D$ is even more convincing in this regard, since the next speaker is starting a new topic, which is novel by definition - at least on the level of subjective self-discovery. If this topic is appreciated by both speakers, who as a result keep the conversation going, it is useful as well and can therefore be considered creative.

In the following section we will introduce the data that we have used to study impasses and to validate our framework. 


\section{METHOD}

Recorded telephone conversations offer the advantage of reducing the influence of body language and para-linguistic information in the conversation, as the interlocutors have to express all the information they want to convey via the audio channel only. Plus, the number of interlocutors is limited to two people in classic phone calls. According to our working hypothesis, this increases the chance of observing an impasse.

For these reasons, we analysed some phone calls in the CallHome database, a publicly available corpus of telephone conversations between speakers of American English (Canavan, Graff, \& Zipperlen, 1997; MacWhinney, 2007). It was developed by the Linguistic Data Consortium (LDC) in support of the project Large Vocabulary Conversational Speech Recognition (LVCSR) and sponsored by the U.S. Department of Defense. The database consists of 120 unscripted telephone conversations up to 30 minutes in length. The calls were recorded in the 1990s, and in exchange for their participation the speakers received free international phone calls for up to 30 minutes. All the calls originated from North America and most of them were directed to a person, usually a friend or relative, located abroad. The corpus is available at http://www.talkbank.org/CABank/.

We used methods from Conversation Analysis (CA) to study how impasses are solved in spontaneous conversations. Intertwined with Pragmatics, Linguistics and Sociology, CA studies the dynamics of conversation, and tries to explain verbal and nonverbal interactions on the basis of the interlocutors' intentions (Sacks, 1992; Schegloff, 2007). For example, a much discussed topic in CA is the study of turn-taking, i.e. the implicit rules according to which it is appropriate (or not) for an interlocutor to start a new turn (Lerner, 2004; Sacks, Schegloff, \& Jefferson, 1974; Stivers et al., 2009). In our model this is partially coded in step A. While an in-depth analysis of conversation dynamics is not required for the current work, we still borrow some techniques and conventions from CA e.g. for transcribing speech - as a useful set of tools described in Jefferson (2004).

For our analysis we randomly selected 13 out of the 120 available phone calls, with a total length of 245 minutes. In one of these conversations the phone was handed over to a third speaker. We treated this as a separate conversation, which brought the total number of analysed conversations to 14 . Two independent raters listened to the phone conversations. They were instructed to identify impasses (stalemates) in the conversation based on [their] subjective rating. In addition, they were asked to assign each part of the conversation to either one of the general domains study, work, or personal as well as identify[ing] meaningful units and write down the general topic. This scoring technique is very similar to the one used for divergent thinking tasks, as described in Wallach and 
Kogan (1965) and Silvia et al. (2008). A change of topic was expected to coincide with an impasse, if interlocutors followed the steps $D$ and $E$ in our model. In addition, we asked our raters to mark speech interruptions and overlaps, i.e. when one speaker started talking in the middle of the other person's turn or when both speakers started speaking at the same time after a pause. These overlaps were intended to help us to identify interruptions in step B in our model.

Below we report the transcriptions of three instances of impasses found in the corpus. Following some standard CA conventions, the number in brackets represents silence time (in seconds) and transcriptions enclosed within square brackets indicate overlapping speech. A colon indicates a prolonged segment in speech while transcriptions such as tch, mm, hhh, indicate, respectively, a click of the mouth, an assertive vocalization, and an out-breath. Finally, the left arrow symbols highlight where in the extract our phenomenon of interest, the impasse, is happening. The transcriptions aim at representing what was actually said, without any attempt at interpreting the utterance or correcting grammatical mistakes that might have been made by the speakers.

All the following examples show that the conversation has supposedly passed the sequential stages at $B$ and $E$ and is now in a situation where both interlocutors are experiencing an impasse at the same time. All three cases result in an unnatural interruption of the conversational flow which was identified by the raters as an impasse.

In the first example two friends, who also work in the same sector, are talking about a conference happening in Seattle later in the year, and are discussing whether they will be attending it and in particular the route the flight is going to follow:

1. S2: That'd be-'f course Chicago wouldn't be: (0.5) .hhh too bad either cos

2. it's (0.4) er:

3. S1: Polar Air (?)

4. S2:(0.4) Yeah (0.3) so it'd be it would be a direct flight

5. S1: $\mathrm{mmh}$

6. (1.8)

7. $\mathrm{S} 1: \mathrm{mh}[\mathrm{h}]$

8. S2: [but]

9. (1.8)

10. S1: well

11. (0.6)

12. S2: We're- hhh. we're g-going home for Christmas

Conversation Analysis 1: CallHome 4112 Conference in Seattle 
After a rather long hesitation, made of silences and filled pauses marked as the phenomenon of interest in lines 4 to 10 , speaker 2 , who also happens to be the last speaker before the impasse, resolves it by completely changing the topic from the conference to the Christmas holidays. This corresponds to the impasse being resolved in step D which in this case also means a change in the domain of the conversation, from work to personal.

In an example from another phone call, two people are talking about how one of them got an evening job in addition to her studies:

1. S2: And then I can work (.) like (0.4) evenins during school

2. S1: (0.3) $A$ [ha ]

3. S2: [I don't (.) ] have to work in the day

4. (1.8) $\leftarrow$

5. S1: tch oh very good $\leftarrow$

6. (1.0)

7. S1: And how's Lee Roy?

Conversation Analysis 2: CallHome 638 New job

While speaker 2 was the last speaker before the impasse marked in lines 4 to 6 , the impasse is resolved by speaker 1. Again, both the topic and the domain are changed, but in addition to the previous example an overlap A between the two speakers occurred (see lines 2 and 3) after which speaker 2 continued to talk as decided in $A$, but then none of them managed to produce a sentence to continue the topic in $\mathrm{C}$.

Their joint impasse finally seems to be resolved by starting a new topic in D. In this final example, two people are talking about holidays around the $4^{\text {th }}$ July:

1. S2: Do you have to work tomorrow?

2. S1: (0.3) Yes

3. S2: (0.3) Did you have hols yesterday?

4. S1: $(0.4)$ Yes

5. S2: (0.3) Oh that's good

6. S1: (0.3) But and it was paid too

7. S2: (0.4) Ohh: is it paid today?

8. S1: $(0.1)$ No

9. S2: $(0.4) \mathrm{Oh}$

10. (1.6)

11. S1: but er:

12. (0.7)

13. S1: Yeah (0.4) So the gym's going well

Conversation Analysis 3: CallHome 4092 Paid holiday time 
In this case, the impasse is resolved by speaker 1 , who was also the last one contributing to the topic in line 8 . Once again the impasse is overcome by a change in domain, from work to personal, but in this case speaker 1 revisits a topic mentioned earlier in the conversation. This would correspond to step E in Figure 1.

Apart from qualitative data, we extracted some quantitative data relative to the speech signal using the software for phonetic analysis Praat (Boersma \& Weenink, 2010). We recorded the total duration of the conversation, the duration of the individual impasses, the identifier of the speaker before and after the impasse, and the time distance between the impasse and the first preceding or following interruption. We also calculated the phonation time of each interlocutor, i.e. the period of time they are speaking without pauses, and the number of syllables. This was obtained counting the nucleus of a syllable by finding voiced peaks in energy surrounded by dips, as described in de Jong and Wempe (2009). Given that each speaker was recorded on a separate audio channel, there is a noticeable difference between the combined phonation time and the sum of the individual times with an overlap of up to $24 \%$ of the combined phonation time. The phonation time was used to identify a dominant speaker within each conversational pair, i.e. the interlocutor who had the longest phonation time. We also calculated the articulation rate, i.e. the quotient of syllable count over phonation time, which is a measure of how fast a speaker talks (see Jacewicz, Fox, O’Neill, \& Salmons, 2009).

\section{RESULTS}

Following the instructions identify impasses (stalemates) in the conversation based on your subjective rating, rater A identified 99 and rater B 118 impasses in the 14 conversations. After their individual assessments the raters were asked to revisit each of the identified impasses to find a shared agreement on its existence and length. As a result, the raters mutually agreed on a total of 110 impasses - on average about 8 per conversation and one every 2 minutes.

All of these identified impasses are accompanied by silence in the conversation, even though the instructions did not explicitly mention this increase in response latency. This observation strengthens the implied overlap between the term impasse, which is commonly used in creativity research, and gaps (silences that can be followed by a change of turn) as described in CA (Sacks, Schegloff, \& Jefferson, 1974).

We also observed nonverbal vocalizations such as mhh, and interjections such as "well" and "yeah" quite frequently during these impasses, as demonstrated in examples for CallHome 4112 and 4092. This is also consistent with previous findings such as in Park (2010), where the adverb anyway is identified as an attempt to move past a conver- 
sational impasse and is often preceded or followed by similar vocalizations. More generally this relates to the role of environmental cues to overcome impasses (e.g. Moss, Kotovsky, \& Cagan, 2011).

The median length of the 110 identified impasses is $m=3.4 s$ with a minimum length of $1.0 \mathrm{~s}$. In the cases where the interlocutors switched to a completely new topic after the impasse, the median length of the impasses is $m=3.5 \mathrm{~s}$; when they resumed a topic that had appeared previously in the conversation, the impasse length is $m=3.9 \mathrm{~s}$.

In 80 out of the 110 identified impasses (72.7\%) the topic changed after the impasse, while in the remaining 30 impasses the interlocutors continued with the same topic as before. While continuing on the same topic does not meet the definition of novel and useful for creative problem solving, the repetitive generation of the same solution can still be perceived as an impasse, as coded by Fleck and Weisberg (2013). Out of the 80 impasses that were followed by a change of topic, 62 (77.5\%, 56.4\% of all impasses) started a topic novel within the conversation, whereas 18 impasses (22.4\%, $16.4 \%$ overall) went back to a topic that had been discussed before. While these 62 changes of topic meet the novelty constraint of a creative solution, revisiting a previous topic can also be interpreted as the repetition of a previous solution (see Fleck \& Weisberg, 2013).

Looking at the 62 conversations that started a novel topic, 36 of them $(58.1 \%$, $32.7 \%$ overall) were resolved by the dominant speaker of that conversation, 22 by the non-dominant speaker (35.5\%, 20.0\% overall), and 4 ended in overlapping speech $(6.5 \%$, $3.6 \%$ overall). Interestingly, in all these overlaps it was the non-dominant speaker who continued talking afterwards. This is also represented by the fact that the dominant speaker was the last speaker before the impasse in 37 cases (59.7\%, 33.6\% overall), while, in the remaining 25 cases (40.3\%, $22.7 \%$ overall), it was the non-dominant speaker. The association between dominance and impasse solving could be related to fluency, which has been shown to be a confounding factor of creativity (Hocevar, 1979; Silvia et al., 2008). However, the fact that dominant speakers spend more time talking means that they have a higher probability of starting a sentence after a gap.

For the 18 impasses that were resolved by revisiting a previously discussed topic, only in 7 cases $(38.9 \%, 6.3 \%$ overall) the dominant speaker was talking before, as opposed to the non-dominant speaker, who spoke in 11 cases (61.1\%, 10.0\% overall). Finally, 11 of these 18 impasses $(61.1 \%, 10.0 \%$ overall) were resolved by the dominant speaker, 6 by the non-dominant one (33.4\%, 5.4\% overall), and 1 (5.6\%, $0.9 \%$ overall) with an overlap - which was won by the dominant speaker.

In total, $54.5 \%$ of the occurrences had a dominant speaker right before the impasse started, and $58.8 \%$ were overcome by a dominant speaker. 


\section{DISCUSSION}

Given the simple instruction of identifying impasses in the conversation corpus, both raters were able to identify a similar number which shows a shared understanding of the task. There was a high overlap between their ratings and they eventually agreed on 110 impasses over 245 minutes of phone calls - which on average translates to about one impasse every two minutes. This confirms our starting argument that the phenomenon which the raters identified as impasses is not only happening inside a conversation, but can also be identified by an observer from the outside. The fact that all the events marked as impasses had an increased response latency additionally supports the idea that the problem solving process can be described using a sequential model (Amabile, 1983; Wallas, 1926). At the same time, this finding ties the observed phenomenon of a gap or lapse in conversation (Sacks, Schegloff, \& Jefferson, 1974) to that of impasse in problem solving (Öllinger, Jones, \& Kloblich, 2014; Weisberg, 2015).

Interestingly there was no impasse with a pause of less than 1 second marked by our raters. While the conversations might have had instances of impasses shorter than this, the raters did not identify them. Also, since pauses, nonverbal vocalizations, and interjections are sometimes used as rhetorical figures of speech, our raters might have identified them as such instead of impasses.

Looking at the length of pauses it is worth noting that impasses followed by a new topic are shorter than the ones where the speakers went back to a previous topic. This suggests that during an impasse speakers might look for a solution in the same order across individuals, potentially even in the same order as depicted in Figure 1: first step $D$ and then $E$. Also, the distribution of impasses that were resolved by a new topic (62), continued on the current one (30), and went back to a previous one (18) supports the idea of a distinct sequence of steps to solve the problem. In this case the continuation on the same topic comes before the revisiting of a previous one which might imply, at least for the case of an impasse, that the order of steps changes from D, C, and E. We did not explore this in more detail as the exact order of steps is not relevant for our argument.

The dominant speakers who are considered to be more active and who spend more time talking, are also more likely to be talking before or after an impasse occurs, thus suggesting that dominant speakers might be more involved in initiating and solving impasses than non-dominant speakers. Again, this supports the correlation between higher fluency and originality in problem solving literature (e.g. Silvia et al., 2008). Interestingly this is not the case for the speakers talking before an impasse which will be resolved with a revisited topic. But this trend might simply be due to random noise in our small sample 
size. Another explanation could be rooted in individual differences in articulation rate: spending more time talking does not necessarily mean that a speaker will be perceived as more active, since she / he might just be speaking more slowly. The mean articulation rate across all the individual speakers in our sample was $A=4.47^{\text {syllables }} / \mathrm{s}$. While the small sample size does not allow any conclusion based on quantitative analysis for the current paper, it might prove useful to observe the following trend in a future study: nondominant speakers seem to be talking more often before impasses that are solved without starting a novel topic. This trend is consistent with the idea that speakers with a low fluency fail to create original sentences which will then lead to the observed impasses.

\section{CONCLUSION}

We are aware that our results can only be understood as a theoretical exploration since observational studies require a large data set to produce reliable quantitative results. Instead, we use the transcriptions and ratings to maintain the possibility that the observed trends can be interpreted as our framework suggests and as is shown in Figure 1. Our framework is consistent with and supports sequential models of creative problem solving, from social creativity, to neuroimaging studies, and behavioural as well as theoretical work (as a representative sample of work, see Bowden \& Jung-Beeman, 2006; Wallach \& Kogan, 1965; Wallas, 1926; Weisberg, 2015). For future work we suggest using a larger sample size which would allow, in a quantitative approach, to draw statistical inference and to apply logistic regression techniques to predict, for example, speaker dominance before and after the impasse from the other available factors (topic, domain, duration, articulation rate, etc.).

Sometimes an impasse within a situation might be provoked intentionally by one of the speakers, for example to signal fatigue or to test the other participants' interest in the current topic. Therefore, a future study could be based on qualitative methods to revisit subjective ethnography and compare collected reports with silently delayed responses and occurrences of non-verbal vocalizations during the impasses.

The preliminary results presented in this paper provide evidence that impasses in conversation exist and are perceivable by external listeners. The impasses analysed in our corpus support the hypothesis that impasses in conversation are resolved by a continuous problem solving sequence of search steps in the solution spaces (new topic, previous topic, etc.). They also provide evidence for the conceptual switching hypothesis. These two models are also described as business-as-usual and the insight-sequence (Newell \& Simon, 1972; Ohlsson, 2011). Our framework is neutral regarding which of these models should be used to describe conversations, and it also conforms with the in- 
tegrated theory proposed in Weisberg (2015). Our suggested framework helps to understand an ongoing conversation as a sequence of time-based events, intertwined with potentially quick, implicit, and distinct steps.

From our reading of the literature as well as the trends observed in this study, we conclude that conversation can be seen as continuous series of creative problem solving. As far as we are aware, this is the first study that links spoken language and creativity through the phenomenon of impasses.

\section{REFERENCES}

Amabile, T. M. (1983). The social psychology of creativity: a componential conceptualization. Journal of Personality and Social Psychology, 45(2), 357-376.

Austin, J. L. (1975). How to do things with words. Oxford University Press.

Beeftink, F., Van Eerde, W., \& Rutte, C. G. (2008). The effect of interruptions and breaks on insight and impasses: do you need a break right now? Creativity Research Journal, 20(4), 358-364.

Boden, M. A. (2004). The Creative Mind: Myths and Mechanisms (2nd edition). Routledge.

Boersma, P. \& Weenink, D. (2010). Praat: doing Phonetics by computer.

Bowden, E. M. \& Jung-Beeman, M. (2006). Methods for investigating the neural components of insight. Methods, 42, 87-99. doi:doi:10.1016/j.ymeth.2006.11.007

Canavan, A., Graff, D., \& Zipperlen, G. (1997). Callhome American English speech. Linguistic Data Consortium.

Chambliss, C. A. \& Feeny, N. (1992). Effects of sex of subject, sex of interrupter, and topic of conversation on the perceptions of interruptions. Perceptual and Motor Skills, 75(3f), 1235-1241.

Clark, H. H. (1996). Using language. Cambridge University Press.

Cranford, E. A. \& Moss, J. (2012). Is insight always the same? a protocol analysis of insight in compound remote associative problems. The Journal of Problem Solving, 4. doi:10.7771/1932-6246.1129

Cummins, F., Li, C., \& Wang, B. (2013). Coupling among speakers during synchronous speaking in English and Mandarin. Journal of Phonetics, 41(6), 432-441.

de Jong, N. H. \& Wempe, T. (2009, May). Praat script to detect syllable nuclei and measure speech rate automatically. Behavior Research Methods, 41(2), 385-390. doi:10.3758/BRM.41.2.385 
Demuth, C. \& Glaveanu, V. P. (2016). Creativity - A New Vocabulary. In V. P. Glaveanu, L. Tanggaard, \& C. Wegener (Eds.), (Chap. Language, pp. 52-60). Palgrave Macmillan. doi:10.1057/9781137511805

Donaldson, S. K. (1979). One kind of speech act: How do we know when we're conversing? Semiotica, 28(3-4), 259-300.

Fischer, G., Giaccardi, E., Eden, H., Sugimoto, M., \& Ye, Y. (2005). Beyond binary choices: Integrating individual and social creativity. International Journal of Human Computer Studies, 63, 482-512. doi:10.1016/j.ijhcs. 2005.04.014

Fleck, J. I. \& Weisberg, R. W. (2013). Insight versus analysis: Evidence for diverse methods in problem solving. Journal of Cognitive Psychology, 25, 436-463. doi:10.1080/20445911.2013.779248

Gilhooly, K. J., Fioratou, E., Anthony, S. H., \& Wynn, V. (2007). Divergent thinking: Strategies and executive involvement in generating novel uses for familiar objects. British Journal of Psychology, 98, 611-625. doi:10.1348/096317907X173421

Grice, H. P. (1957). Meaning. The Philosophical Review, 377-388.

Grice, H. P. (1975). Syntax and semantics: Speech acts Volume 3. In P. Cole \& J. L. Morgan (Eds.), (Chap. Logic and conversation. pp. 41-58). New York: Academic Press.

Guilford, J. P. (1967). The Nature of Human Intelligence. McGraw-Hill.

Halliday, M. A. K. (1994). An Introduction to Functional Grammar. London: Edward Arnold.

Hocevar, D. (1979). Ideational Fluency as a Confounding Factor in the Measurement of Originality. Journal of Educational Psychology, 71(2), 191-196.

Howard, T., Culley, S., \& Dekoninck, E. (2007). Creativity in the Engineering Design Process. In International conference of engineering design.

Jacewicz, E., Fox, R. A., O'Neill, C., \& Salmons, J. (2009, July). Articulation rate across dialect, age, and gender. Language Variation and Change, 21(02), 233-256. doi:10.1017/s0954394509990093

Jefferson, G. (2004). Conversation analysis: studies from the first generation. In G. H. Lerner (Ed.), (Chap. Glossary of transcript symbols with an introduction, pp. 13-31). John Benjamins Publishing.

Jordan, M. E., Lanham, H. J., Crabtree, B. F., Nutting, P. A., Miller, W. L., Stange, K. C., \& McDaniel, R. R. (2009). The role of conversation in health care interventions: Enabling sensemaking and learning. Implement Sci, 4(15), 1-13.

Kaplan, M. A. (1997). Learning to converse in a foreign language: The reception game.

Simulation \& Gaming, 28(2), 149-163. 
Kaufman, J. C. \& Beghetto, R. A. (2013). Do people recognize the four Cs? Examining layperson conceptions of creativity. Psychology of Aesthetics, Creativity, and the Arts, 7(3), 229-236. doi:10.1037/a0033295

Lerner, G. H. (2004). Conversation analysis: Studies from the first generation. John Benjamins Publishing.

MacWhinney, B. (2007). The Talkbank Project. In Creating and Digitizing Language Corpora (pp. 163-180). Springer.

McCarthy, M. (2001). The Cambridge Guide to Teaching English to Speakers of Other Languages. In D. Nunan \& R. Carter (Eds.), (Chap. Discourse, pp. 48-55). Cambridge: Cambridge University Press.

Moss, J., Kotovsky, K., \& Cagan, J. (2011). The effect of incidental hints when problems are suspended before, during, or after an impasse. Journal of Experimental Psychology: Learning, Memory and Cognition, 37(1), 140-148. doi:10.1037/a0021206

Newell, A. \& Simon, H. A. (1972). Human Problem Solving. Prentice-Hall International, Inc.

Ohlsson, S. (2011). Deep Learning: How the Mind Overrides Experience. Cambridge University Press.

Öllinger, M., Jones, G., \& Knoblich, G. (2014). The dynamics of search, impasse, and representational change provide a coherent explanation of difficulty in the ninedot problem. Psychological Research, 78(2), 266-275.

Park, I. (2010). Marking an impasse: The use of anyway as a sequence-closing device. Journal of Pragmatics, 42. doi:10.1016/j.pragma.2010.06.002

Reed, S. K. (2015). The Structure of III-Structured (and Well-Structured) Problems Revisited. Educational Psychology Review, 1-26. doi:10.1007/s10648-0159343-1

Runco, M. A. \& Jaeger, G. J. (2012). The Standard Definition of Creativity. Creativity Research Journal, 24(1), 92-96. doi:10.1080/10400419.2012.650092

Sacks, H. (1992). Lectures on Conversation, Volumes I and II (G. Jefferson, Ed.). Oxford: Basil Blackwell.

Sacks, H., Schegloff, E. A., \& Jefferson, G. (1974). A simplest systematics for the organization of turntaking for conversation. Language, 696-735.

Salvi, C., Bricolo, E., Kounios, J., Bowden, E., \& Beeman, M. (2016). Insight solutions are correct more often than analytic solutions. Thinking \& Reasoning. doi:10. 1080/13546783.2016.1141798

Schegloff, E. A. (2007). Sequence organization in interaction: Volume 1: A primer in conversation analysis. Cambridge University Press. 
Searle, J. R. (1969). Speech acts: An essay in the philosophy of language. Cambridge University Press.

Selting, M. (1996). On the interplay of syntax and prosody in the constitution of turnconstructional units and turns in conversation. Pragmatics, 6, 371-388.

Silvia, P. J., Winterstein, B. P., Willse, J. T., Barona, C. M., Cram, J. T., Hess, K. I., ... Richard, C. A. (2008). Assessing creativity with divergent thinking tasks: Exploring the reliability and validity of new subjective scoring methods. Psychology of Aesthetics, Creativity, and the Arts, 2(2), 68-85. doi:doi:10.1037/1931-3896. 2.2.68

Simon, H. A. (1973). The Structure of III Structured Problems. Artificial Intelligence, 4, 181-201.

Stein, M. I. (1953). Creativity and Culture. The Journal of Psychology: Interdisciplinary and Applied, 36(2), 311-322. doi:10.1080/00223980.1953.9712897

Stivers, T., Enfield, N. J., Brown, P., Englert, C., Hayashi, M., Heinemann, T., ... Yoon, K. -E., et al. (2009). Universals and cultural variation in turn-taking in conversation. Proceedings of the National Academy of Sciences, 106(26), 10587-10592.

Wallach, M. A. \& Kogan, N. (1965). Modes of Thinking in Young Children. A Study of the Creativity-Intelligence distinction. Holt, Rinehart, and Winston, Inc.

Wallas, G. (1926). The Art of Thought. Jonathan Cape London.

Warren, M. (2006). Features of Naturalness in Conversation. Pragmatics \& Beyond New Series. John Benjamins Publishing. doi:10.1075/pbns.152

Weisberg, R. W. (2015). Toward an integrated theory of insight in problem solving. Thinking \& Reasoning, 21(1), 5-39. doi:10.1080/13546783.2014.886625

Corresponding author at: Ilaria Torre, CogNovo, Link3, Plymouth University, Drake Circus, PL4 8AA, Plymouth, UK.

E-mail: Ilaria.Torre@ Plymouth.ac.uk 